\section{A) Check for updates}

Cite this: Food Funct., 2020, 11, 3916

\title{
The effect of quercetin on endothelial cells is modified by heterocellular interactions
}

\author{
Sarka Tumova, (DD a,b Michael J. Houghton (D) a,c and Gary Williamson (D) *a,c
}

Single cell-type models are useful for determining mechanisms, but in vivo, cell-cell interactions are important, and neighbouring cells can impact endothelial cell function. Quercetin can attenuate endothelial dysfunction by modulating vascular tone and reducing inflammation. We determined the effect of quercetin on a co-culture between Human Umbilical Vein Endothelial Cells (HUVEC) and human HepG2 hepatic cells or human LHCN-M2 muscle cells. Heme oxygenase-1 (HO-1) mRNA and protein were decreased, pyruvate dehydrogenase kinase (PDK) 4 and glucose transporter (GLUT) 3 mRNA increased, and GLUT1 protein decreased in HUVEC when cultured with HepG2. GLUT transporters, but not the other targets, were similarly regulated in co-culture with muscle cells. Some but not all of the effects were mediated by lactate and transforming growth factor $\beta 1$. Quercetin added apically to the endothelial cells upregulated $\mathrm{HO}-1$ and downregulated PDK4 both in monoculture and in co-culture, but the total PDK4 levels were higher in the presence of HepG2 cells. In the absence of general permeability changes, glucose transport across the endothelial monolayer was elevated in the presence of HepG2 cells, however this effect was moderated by quercetin applied on the apical side of the endothelial cells. At lower glucose concentration, apical quercetin also promoted glucose uptake in HepG2 cells. Co-culturing HUVEC with the HepG2 cells showed capacity to modulate quercetin-elicited changes in endothelial gene transcription and glucose transport.

Received 15th January 2020 Accepted 18th March 2020

DOI: 10.1039/d0fo00141d

rsc.li/food-function culating cells and molecules with the feedback from the adjacent tissues in order to secure adequate supply of nutrients and provide capacity for efficient organ maintenance and function. ${ }^{8}$ The context of the nascent environment can have a significant impact and is indispensable for endothelium-specific phenomena such as the nitric oxide (NO) mediated vasorelaxation, where the signalling between endothelial and smooth muscle cells in larger vessels is critical for the regulation of blood pressure. ${ }^{9}$ Similarly, the presence of astrocytes and pericytes is critical for blood-brain barrier integrity and maintenance. ${ }^{10}$ The communication between neighbouring cell types in capillaries can regulate endothelial cell proliferation and angiogenesis, and control access of growth factors and hormones to the tissues, as demonstrated for delivery of insulin to the skeletal muscle ${ }^{11,12}$ or modulation of hepatic regeneration and fibrosis. ${ }^{13}$ At the same time, interactions with cells in underlying tissues can modulate the effects of circulating molecules on the endothelial cells.

Diet-derived small molecules such as polyphenols and their metabolites that circulate in the blood affect various aspects of endothelial function post-prandially. Intervention and epidemiological studies have found positive association of polyphenol-rich food with cardiovascular health benefits, including decrease in inflammatory markers and improved blood pressure. ${ }^{14-16}$ Further in vitro and animal studies attributed 
these benefits to pathways that improve redox balance and reduce inflammation, ${ }^{17}$ specifically via modulating endothelial nitric oxide synthase (eNOS) function, inhibiting NADPH oxidases (NOX) and other reactive oxygen species (ROS)-generating enzymes and stimulation of nuclear factor erythroid 2-related factor 2 (Nrf2) pathways. ${ }^{18,19}$ One of the best studied polyphenols is the flavonoid quercetin, with specific protective effects on the vasculature described in animal and in vitro studies. It can alleviate some consequences of atherogenic and inflammatory signalling, including endothelial damage induced by oxidized low-density lipoprotein (oxLDL) or angiotensin II, by activating SIRT1 and modulating NOX/eNOS activities, ${ }^{20}$ or by decreasing NOX subunit. ${ }^{21,22}$ In the ApoE-/mouse model, quercetin attenuates atherosclerosis and improves NO bioavailability through heme oxygenase 1 (HO-1) regulation. ${ }^{23}$ It also improves redox balance by regulating oxidation, conjugation and export of glutathione in human aortic endothelial cells. ${ }^{24}$ Quercetin regulates blood pressure in rats and humans via local deconjugation of the plasma metabolite quercetin glucuronide. $^{25,26}$ Quercetin can also downregulate adhesion molecules that attract monocytes during inflammation, such as vascular cell adhesion molecule 1 (VCAM1) or intercellular adhesion molecule 1 (ICAM1), ${ }^{27,28}$ and decrease attachment of monocytes to endothelial cells via Nrf2 activation..$^{29}$ We have reported recently that quercetin can affect endothelial cell metabolism through hypoxia induced factor $1 \alpha$ (HIF1 $\alpha)$ and the Nrf2 pathway. ${ }^{30}$ However, despite the fact that the endothelium in vivo functions as a part of a larger system, most of the in vitro models rely on single-type cell cultures.

In order to examine the impact of other cells on quercetin modulation of endothelial function to better mimic in vivo conditions, here we have used HUVEC as a model in noncontact co-cultures and studied the endothelial gene expression and glucose uptake and transport with and without quercetin. HUVEC were cultured in the presence of HepG2 cells or immortalized primary skeletal muscle cells to elucidate the impact on selected gene expression, and then the effects of plant-derived polyphenol quercetin on HUVEC were examined in mono-culture and co-culture with the carcinoma cells.

\section{Methods}

\subsection{Cell culture and co-culture}

HUVEC (Lonza Sales Ltd, Switzerland) were cultured in endothelial growth medium 2 (EGM-2) supplemented with EGM-2 BulletKit (Lonza) and used between passage 3-6. HepG2 cells (ATCC cat\# HB-8065, LGC Promochem, Teddington, UK) were cultured in Eagle's Minimum Essential Medium supplemented with $10 \%(\mathrm{v} / \mathrm{v})$ heat-inactivated fetal bovine serum (FBS), $100 \mathrm{U}$ $\mathrm{ml}^{-1}$ penicillin, $100 \mathrm{mg} \mathrm{ml}^{-1}$ streptomycin, $2 \%$ (v/v) non-essential amino acids, and $1 \%(\mathrm{v} / \mathrm{v})$ sodium pyruvate (all from Sigma-Aldrich, Gillingham, UK). Muscle cells were immortalized skeletal muscle cells, LHCN-M2, a gift of Dr Vincent Mouly (Sorbonne Université, INSERM, Institute of Myology,
Centre of Research in Myology, Unite Mixte de Recherche Scientifique 974, Paris, France). ${ }^{31}$ They were cultured in Dulbecco's Modified Eagle Medium (DMEM) (Gibco, Life Technologies, ThermoFisher, UK) supplemented with $5.5 \mathrm{mM}$ glucose, $10 \%(\mathrm{v} / \mathrm{v})$ FBS (heat-inactivated), 10\% v/v heat-inactivated newborn calf serum (Gibco), hEGF (10 $\mu \mathrm{g} \quad \mathrm{L}^{-1}$; PeproTech, London, UK), hbFGF (1 $\mu \mathrm{g} \mathrm{L}^{-1}$; Gibco) and dexamethasone $\left(400 \mu \mathrm{g} \mathrm{L}^{-1}\right)$ (Sigma), as described previously. ${ }^{32}$ Myocytes were differentiated upon confluency by switching to a differentiation medium (5.5 mM glucose in DMEM, $2 \%$ horse serum (Gibco)) for up to 4 days. ${ }^{32}$ For the co-cultures, HUVEC were seeded on semi-permeable membrane supports in 6-well $\left(2 \times 10^{5}\right.$ cells per well) Transwell plates (Corning, New York, USA). After 3 days, membrane inserts with the HUVEC monolayers were transferred on top of wells in 6-well plates with nearly confluent HepG2 cells or 3-day differentiated LHCN-M2 muscle cells, using $0.2 \%$ FBS in EGM- 2 as the basal and apical medium, and the cells were co-cultured for a further $24 \mathrm{~h}$. Alternatively, after $1 \mathrm{~h}$ incubation, the medium on apical side was replaced with fresh $0.2 \%$ FBS in EGM-2 with $5 \mu \mathrm{M}$ quercetin or $0.01 \%$ DMSO and the cells were further incubated for 18 hours. At the end of the co-culture, monolayer trans-endothelial electrical resistance (TEER) was measured using a Millicell ERS voltohmmeter (Millipore (UK) Ltd, Watford, UK) according to the manufacturer's instructions. After that, the cells were harvested or the glucose uptake assays were performed as described below. The quercetin (Extrasynthese, Genay, France) stock was prepared at $50 \mathrm{mmol}$ $\mathrm{L}^{-1}$ in DMSO and diluted to the final concentration of $5 \mu \mathrm{mol}$ $\mathrm{L}^{-1}$ for the treatments. DMSO was used at $0.01 \%$ in the corresponding control. For TGF $\beta 1$ treatment, human recombinant TGF 1 (Sigma) was applied to the cells in the EGM-2 medium at $50 \mathrm{ng} \mathrm{ml}{ }^{-1}$.

\subsection{Glucose uptake and transport}

Cells were washed with glucose-free buffer $\left(2.5 \mathrm{mM} \mathrm{MgCl}_{2}\right.$, $1 \mathrm{mM} \mathrm{CaCl}_{2}, 0.14 \mathrm{M} \mathrm{NaCl}, 20 \mathrm{mM}$ HEPES, 3.5\% $\mathrm{NaHCO}_{3}, \mathrm{pH}$ 7.3), incubated for $10 \mathrm{~min}$ in glucose-free RPMI medium and then supplemented for $10 \mathrm{~min}$ with $5.5 \mathrm{mM} \mathrm{D}-\left[{ }^{14} \mathrm{C}(\mathrm{U})\right]$-glucose $\left(0.1 \mu \mathrm{Ci} \mathrm{ml}{ }^{-1}\right)$ (PerkinElmer, Seer Green, UK) on the apical side. Radioactive medium on the apical and basolateral side was collected and the radioactivity measured by scintillation counting. The cells were washed three times with cold PBS with $\mathrm{Ca}^{2+}$ and $\mathrm{Mg}^{2+}$, lysed in $1 \mathrm{M} \mathrm{NaOH}$ and radioactivity in the cell lysate aliquot, neutralized with $1 \mathrm{M} \mathrm{HCl}$, was determined by scintillation counting. Measured radioactivity was converted to glucose concentration using standard calibration curves constructed using medium with known amounts of ${ }^{14} \mathrm{C}$ labelled glucose. Small aliquots of cell lysates were used to measure protein content by Coomassie Protein Assay (Pierce, Thermo Fisher Scientific, Altrincham, UK) and uptake corrected for protein content.

\section{3. mRNA quantification by droplet digital PCR (ddPCR)}

The ddPCR was performed using cDNA obtained by reverse transcription (high capacity RNA-to-cDNA kit, Life 
Technologies) from mRNA, isolated from cell lysates (AurumTM-Total RNA Mini kit, Bio-Rad Laboratories Ltd, Herts, UK) as recommended by the standard manufacturer's protocols. Guidelines for digital $\mathrm{PCR}^{33}$ were followed as appropriate. FAM-labelled gene-specific probes were from Bio-Rad: glucose transporter (GLUT)1 (SLC2A1), dHsaCPE5035286; and Life Technologies: GLUT3 (SLC2A3), Hs00359840_m1; GLUT6 (SLC2A6), Hs01115485_m1; HO-1, Hs01110250_m1; ATP binding cassette subfamily $\mathrm{B}$ member 1 (ABCB1) Hs00184500_m1; ATP binding cassette subfamily C member 2 (ABCC2), Hs00166123_m1; ATP binding cassette subfamily G member 2 (ABCG2), Hs01053790_m1; monocarboxylate transporter (MCT)1 (SLC16A1), Hs00161826_m1; MCT4 (SLC16A3), Hs00358829_m1; MCT5 (SLC16A4), Hs00190794_m1; pyruvate dehydrogenase kinase (PDK) 2, Hs00176865_m1; PDK4, Hs01037712_m1. TATA binding protein (TBP) was used as a reference for mRNA quantification using VIC-labelled probe (TBP, Hs00427620_m1, Life Technologies). Samples corresponding to 10-15 ng mRNA were analysed for specific gene expression using QX100 Droplet Digital PCR system (Bio-Rad) as described previously. ${ }^{34}$ Briefly, the PCR reaction mix with the gene-specific fluorescent probes was dispersed into oil droplets using QX-100 Droplet Generator. After 40 cycles on the X1000 Touch, droplets were analysed by QX100 Droplet Reader. QuantaSoft software (Kosice, Slovakia) was used to analyse the data and determine the number of copies present in the mix, which were then expressed as the relevant mRNA/ TBP ratio, or converted to copies per ng of mRNA, assuming $1: 1$ RT PCR efficiency.

\subsection{Protein detection by simple western}

GLUT1, GLUT3, HO-1 and Galectin1 protein levels were detected by automated capillary western blotting on WES (ProteinSimple, Bio-Techne, San Jose, CA, USA). At the end of the co-culture, HUVEC on filters were washed in cold PBS and lysed in $60 \mathrm{mM}$ octyl-glucoside, $150 \mathrm{mM} \mathrm{NaCl}, 20 \mathrm{mM}$ Tris, $\mathrm{pH}$ 7.4 with protease inhibitors. Standard conditions for the western protocol were followed as recommended by the manufacturer, except the incubation with the primary antibodies was extended to $60 \mathrm{~min}$. Rabbit antibodies against GLUT1 (dilution 1:50; Abcam, cat\# ab115730, RRID:AB_10903230) and GLUT3 (dilution 1:100; Abcam, cat\# ab191071, RRID: AB_2736916) were used with rabbit anti-Na,K-ATPase (dilution 1:50; Cell Signaling Technology, New England Biolabs (UK) Ltd, Hitchin, UK, cat\# 3010 RRID:AB_2060983) for a loading control, with denaturation performed for $20 \mathrm{~min}$ at $37^{\circ} \mathrm{C}$ to prevent aggregation. Goat anti-HO-1 $\left(20 \mu \mathrm{g} \mathrm{ml} \mathrm{m}^{-1}\right.$, R\&D Systems, Bio-Techne, cat\# AF3776, RRID:AB_2295362) was used for detection with anti-calnexin $\left(\begin{array}{llll}8 & \mu \mathrm{g} & \mathrm{ml}^{-1}\end{array}\right.$, Santa Cruz Biotechnology, Dallas, TX, USA, cat\# sc-6465, RRID: $\left.\mathrm{AB} \_2069146\right)$ as the loading control and the standard denaturation protocol for $5 \mathrm{~min}$ at $95{ }^{\circ} \mathrm{C}$ was followed for the sample preparation. Galectin-1 was detected with mouse monoclonal antibody (dilution 1:20; Novus Biologicals, BioTechne, cat\# MAB1152, RRID:AB_2811716) using rabbit anti$\alpha$-actinin D6F6 antibody (1:50; Cell Signaling Technology,
NEB, cat\# 6487, RRID:AB_11179206) as a control. Incubation with anti-goat-IgG-HRP (dilution 1:25; R\&D Systems), anti-rabbit-IgG-HRP (ProteinSimple, Bio-Techne), or antimouse-IgG-HRP with 20× anti-rabbit-IgG-HRP (ProteinSimple, Bio-Techne) followed by luminol/peroxide was used to generate the chemiluminescent signal for quantification. All sets of antibodies gave a linear response in the range between 0.1-0.4 mg protein per $\mathrm{ml}$ and $0.2 \mathrm{mg} \mathrm{ml}^{-1}$ was selected for the GLUT and Galectin-1 analysis, while $0.4 \mathrm{mg} \mathrm{ml}^{-1}$ was used for HO-1.

\subsection{TGFß1 ELISA}

Nearly confluent HepG2 cells or 3-days differentiated LHCN-M2 cells were incubated with $0.2 \%$ FBS in EGM-2 medium for $19 \mathrm{~h}$. Conditioned medium was centrifuged for $10 \mathrm{~min}$ at $600 \mathrm{~g}$ and aliquots were stored frozen at $-80{ }^{\circ} \mathrm{C}$ until use. Total secreted TGF $\beta 1$ was quantified after activation of the latent form, using Dual TGF $\beta 1$ Quantikine ELISA (R\&D Systems, Bio-Techne) according to the manufacturer's instructions. The EGM-2 medium with $0.2 \%$ FBS, which contained less than $31 \mathrm{pg} \mathrm{ml}^{-1}$ TGF $\beta 1$, was used as a blank. The measured values were within the linear range of the calibration curve (31-1000 $\mathrm{pg} \mathrm{ml}^{-1}$ ) for the recombinant TGF $\beta 1$ provided by the manufacturer.

\subsection{Lactate quantification}

Lactate in the cell medium was detected and quantified by High Performance Anion Exchange Chromatography with Pulsed Amperometric Detection (HPAE-PAD) using the Dionex ICS-4000 Ion Chromatography System (Thermo Scientific). After treatments, medium was removed from the cell monolayers and centrifuged at $1000 \mathrm{~g}$ to remove cellular debris. Cellfree medium was deproteinized by adding an equal volume of ACN, incubating on ice for $5 \mathrm{~min}$ and centrifuging for $10 \mathrm{~min}$ at $18000 \mathrm{~g}$ at $4{ }^{\circ} \mathrm{C}$. The sample was diluted 50 -fold in MilliQ water to fall within the linear range of lactate

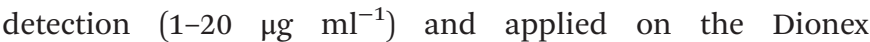
IonPac AS11 (Thermo Scientific) anion exchange column, equilibrated in $1 \mathrm{mM} \mathrm{KOH}$, and a 1-60 $\mathrm{mM} \mathrm{KOH}$ gradient was used for elution. A standard calibration curve was obtained from linear range of concentrations of ICE standard lactate (Sigma).

\subsection{Data analysis}

Test and control data obtained in pairs were compared using independent Student $t$-test. ANOVA with Bonferroni post-hoc analysis was used for sets with multiple samples. Differences with $p<0.05\left(^{*}\right)$ were considered significant. All results were obtained from at least 2-3 independent experiments. Origin software (OriginLab Corporation, Northampton, MA, USA) was used for data analysis and the data are presented as mean \pm SEM. 


\section{Results}

3.1 Co-culturing HUVEC with HepG2 or muscle cells affects differently endothelial gene expression

To establish the impact of HepG2 cells on endothelial cell expression of glucose, anion and drug transporters and metabolism-related genes, confluent HUVEC on Transwell inserts were cultured for $24 \mathrm{~h}$ with or without HepG2 cells on their basolateral side. This affected the mRNA expression of several endothelial genes (Fig. 1A), including significant increases in GLUT3 (236 $\pm 32 \%$ of the control) and PDK4 $(229 \pm 21 \%$ of the control), and decreases in GLUT6, MCT1 and HO-1 (66.7 \pm $15.7 \%, 91.0 \pm 1.5 \%$ and $29.7 \pm 4.5 \%$ of the control, respectively), while the expression of the other tested genes remained unchanged. The downregulation of HO-1 was confirmed at the protein level (72.9 $\pm 7.7 \%$ of the control) (Fig. 1B), but the upregulation of GLUT3 mRNA did not translate to a significant increase in protein (Fig. 1B). On the other hand, protein levels of endothelial GLUT1 were almost halved $(52.5 \pm 4.8 \%$ of the control) in the co-culture even in the absence of mRNA changes (Fig. 1B), indicating involvement of posttranscriptional regulation. Galectin-1 expression has been previously

A

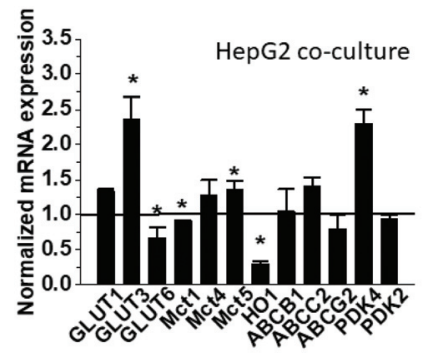

B

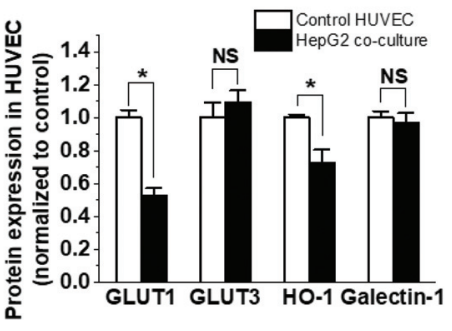

C

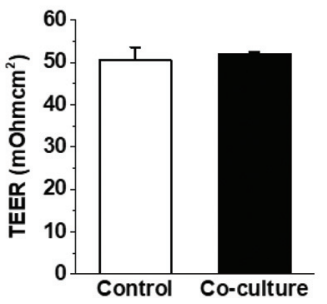

Fig. 1 Gene expression in HUVEC co-culture with HepG2 cells. Confluent HUVEC were cultured on Transwells alone (control) or in the presence of confluent HepG2 cells on the basolateral side (HepG2 coculture) in BulletKit-supplemented EGM-2 medium. After $24 \mathrm{~h}$, the mRNA expression was analysed by $\operatorname{ddPCR}(\mathrm{A})$ and the protein expression for selected genes was determined using automated capillary Western analysis (B). The monolayer integrity was verified by TEER measurement (C). NS, not significantly different. reported to change in endothelial co-cultures, but galectin-1 protein levels were similar between the HUVEC monoculture and co-culture (Fig. 1B). Some of the observed effects were specific for HepG2 cells, as HUVEC co-culture with differentiated skeletal muscle cells affected endothelial mRNA and protein levels differently (Fig. 2A and B). GLUT3 mRNA was still significantly increased in the co-culture $(183 \pm 18.5 \%$ of the control), but in contrast to the HepG2 co-cultures, GLUT6, MCT1, PDK4 and HO-1 mRNA remained unaffected, while GLUT1 mRNA was downregulated by about $20 \%$ (80.9 $\pm 7.9 \%$ of the control). At the protein level, similar to the effect on HepG2 cells, the co-incubation with muscle cells triggered GLUT1 downregulation $(63.1 \pm 3.0 \%$ of the control), while GLUT3 and galectin-1 were not affected. However, in contrast to the HepG2, muscle cells did not affect the endothelial HO-1 protein levels (Fig. 2B).

\subsection{The effects of HepG2-conditioned media on HUVEC gene expression}

One of the most prominent features of HepG2 metabolism is lactate production. In the current setting, the lactate concentration in the media was measured by HPAE-PAD chromato-

A

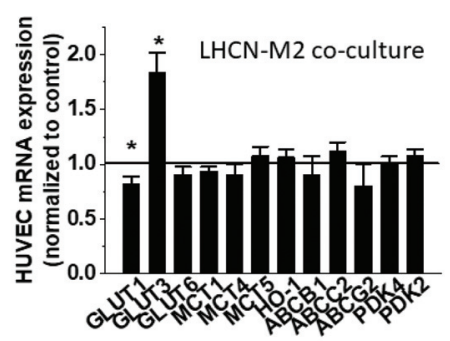

B

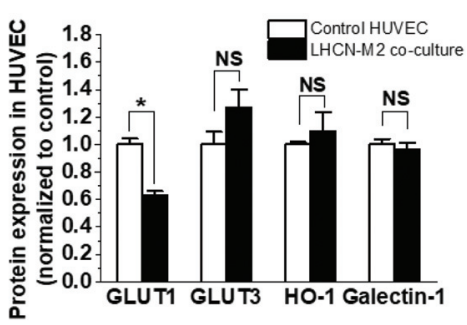

C

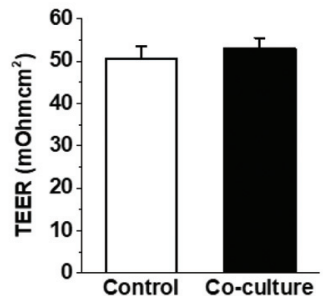

Fig. 2 Gene expression in HUVEC co-culture with muscle cells. Confluent HUVEC were cultured on Transwells alone (control) or in the presence of differentiated LHCN-M2 cells on the basolateral side (LHCN-M2 co-culture) in 0.2\% FBS EGM-2 medium. After $19 \mathrm{~h}$, the mRNA expression was analysed by $\operatorname{ddPCR}(\mathrm{A})$ and the protein expression for selected genes was determined using automated capillary Western analysis (B). The monolayer integrity was verified by TEER measurement (C). NS, not significantly different. 

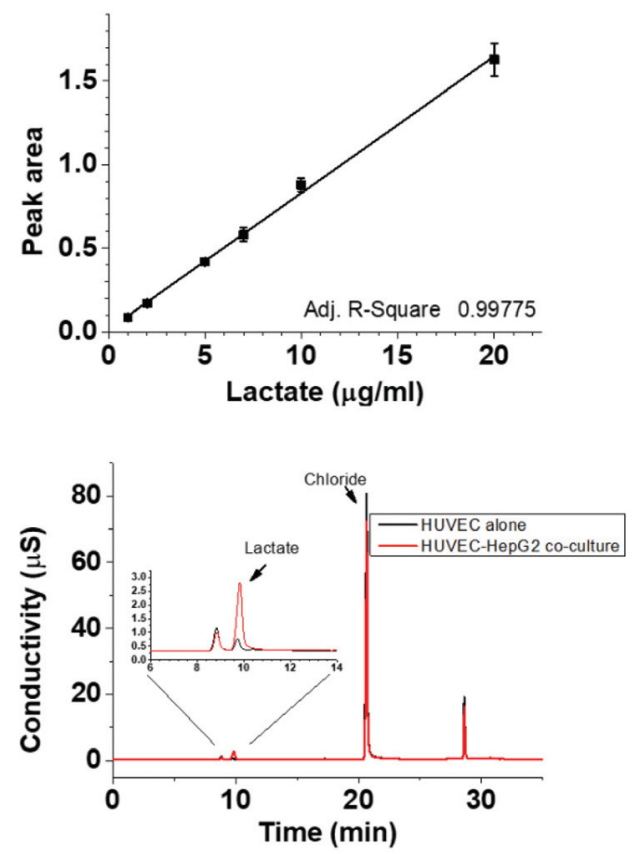

Fig. 3 Extracellular lactate concentration in the HUVEC cultures with and without HepG2 cells. Lactate detection by ICS-4000 ion capillary system was linear in the range between 1-20 $\mu \mathrm{g} \mathrm{ml}^{-1}$ (A). Lactate was detected in 100-fold diluted basolateral media from HUVEC cultured alone or in the presence of HepG2 cells for $24 \mathrm{~h}$ (B). Lactate concentration in undiluted co-culture medium was estimated to be $8.3 \pm$ $0.3 \mathrm{mM}$, while the lactate concentration produced in HUVEC monoculture was $1.1 \pm 0.1 \mathrm{mM}$.

graphy and was estimated to reach $8.3 \pm 0.3 \mathrm{mM}$ at the end of the $24 \mathrm{~h}$ co-culture (Fig. 3), a significant increase compared to $1.1 \pm 0.1 \mathrm{mM}$ lactate measured in corresponding HUVEC monoculture. To test whether the additional lactate or other factors secreted by HepG2 cells into the basolateral medium could be responsible for the observed gene expression changes, HUVEC were cultured for $18 \mathrm{~h}$ in the presence of $8 \mathrm{mM}$ lactate, HepG2-conditioned medium, or HepG2 cells on the basolateral side and the mRNA for MCT1, PDK4 and HO-1 was detected by ddPCR (Fig. 4A, B and C). A small effect of lactate or conditioned media was observed on PDK4 and HO-1 mRNA expression (Fig. 4B and C), but did not reach the magnitude of the changes triggered by HepG2 cell co-culturing. The time-course of the mRNA expression for MCT1, PDK4 and HO-1 was further followed in HUVEC incubated with $8 \mathrm{mM}$ lactate for 1, 3, 6 and $24 \mathrm{~h}$ (Fig. 5). Lactate transiently decreased PDK4 and MCT1 mRNA levels while HO-1 mRNA declined steadily with maximum decrease of $\sim 30 \%$ after $24 \mathrm{~h}$. This indicates that time-dependent changes in lactate concentration may contribute to MCT1 and HO-1 regulation, but are not solely responsible for the full effect.

\subsection{The role of TGFß1 secreted by HepG2 cells in the HO-1 downregulation}

One of the few known negative regulators of HO-1 expression is TGF $\beta 1 .^{35}$ In agreement, incubation with human recombi-
A

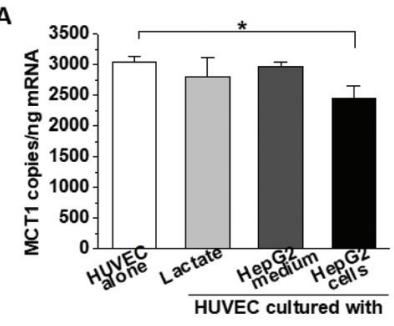

B

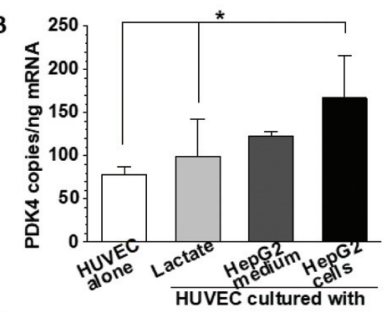

C

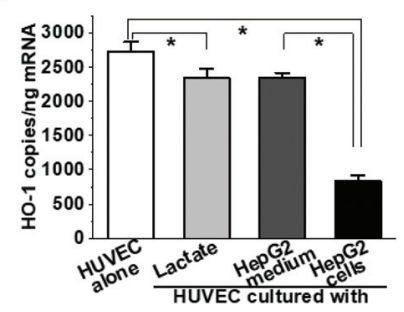

Fig. 4 Effect of culture conditions on gene expression. HUVEC were cultured in the presence of $8 \mathrm{mM}$ lactate, conditioned HepG2 media or HepG2 cells. After $18 \mathrm{~h}$, the expression of MCT1 (A), PDK4 (B) and HO-1 (C) mRNA was determined by ddPCR.
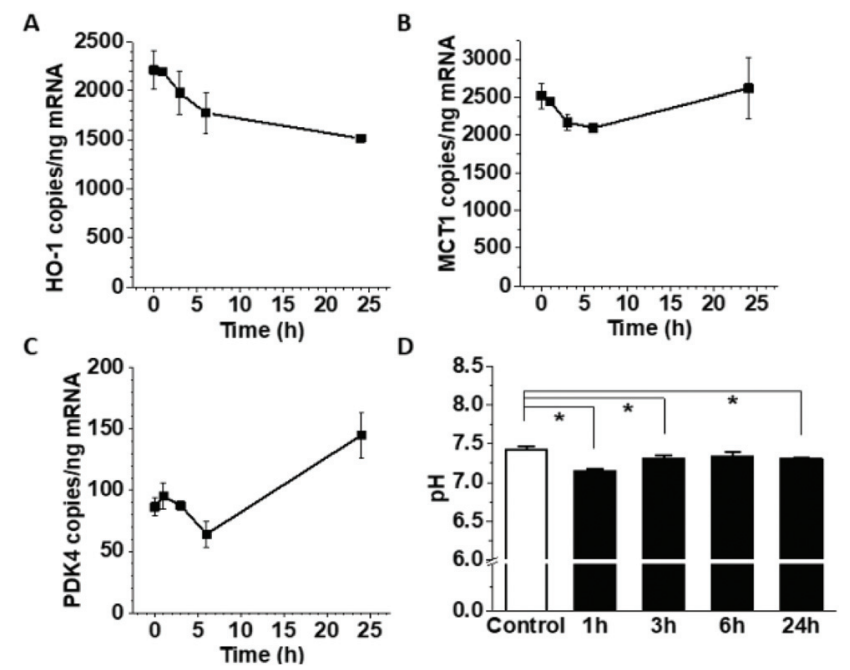

Fig. 5 The effect of pH/lactate on gene expression in HUVEC. Confluent HUVEC on Transwells were exposed to $8 \mathrm{mM}$ lactate on the basolateral side for 1, 3, 6 and $24 \mathrm{~h}$ and mRNA for HO-1 (A), MCT1 (B), and PDK4 (C) was measured by ddPCR. The $\mathrm{pH}$ was monitored after lactate addition and compared to untreated control medium (D).

nant TGF $\beta 1$ decreased HO-1 mRNA expression in HUVEC (Fig. 6A). HepG2 cells secreted a significant amount of TGF $\beta 1$ in the media (Fig. 6B), which could contribute to HO-1 down- 
A

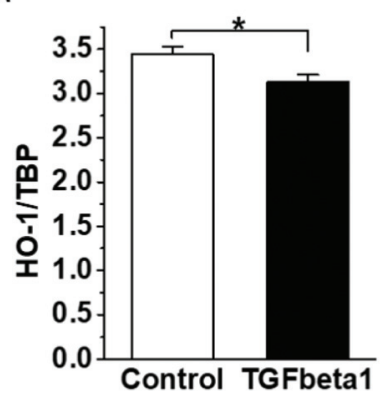

B

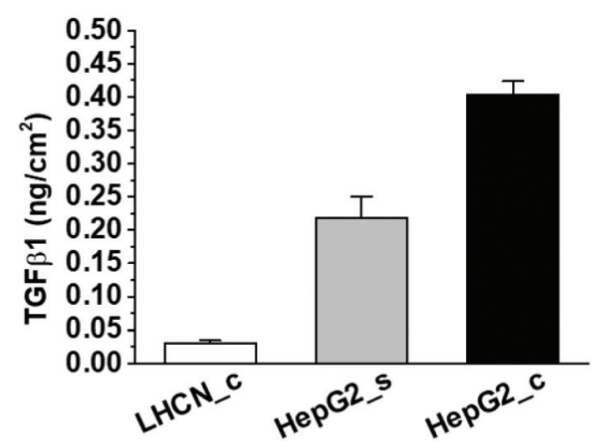

Fig. 6 TGF $\beta 1$ and HO-1 expression in HUVEC. Confluent HUVEC were incubated with recombinant TGF $\beta 1$ for $24 \mathrm{~h}$ and the HO-1 expression was analysed by ddPCR (A). TBP was used for normalization. TGF $\beta 1$ ELISA was used to measure the growth factor secretion in confluent LHCN-M2 muscle cells (LHCN-M2_C) and subconfluent (HepG2_s) and confluent (HepG2_c) HepG2 cells (B). The growth factor secretion was normalized to the cell area.

regulation. Considerably lower amounts of TGF $\beta 1$ were detected in the muscle-conditioned media, consistent with their lack of effect on HO-1 expression in HUVEC.

\subsection{Effects of quercetin on HO-1 and PDK4 expression in HUVEC in the presence and absence of HepG2 cells}

We have shown previously in HUVEC that incubation with quercetin dramatically upregulated HO-1 mRNA at $4 \mathrm{~h}$ while decreasing PDK4 mRNA expression at 18 h. ${ }^{30}$ As both genes were significantly affected by co-culturing with HepG2 cells, we have tested the impact of quercetin in the co-culture. Quercetin was applied to the apical side of HUVEC during the culture in the presence and absence of HepG2 cells and the mRNA for HO-1 and PDK4 was analysed after 4 or $18 \mathrm{~h}$. Compared to untreated cultures, incubation with quercetin for $4 \mathrm{~h}$ significantly upregulated HO-1 mRNA in both monoculture $(160 \pm 11 \%)$ and co-culture $(214 \pm 35 \%)$ (Fig. 7A), consistent with the previous work. ${ }^{30}$ In contrast to the longer, $24 \mathrm{~h}$ incubation (Fig. 1A and 4C), there was no significant effect of coculturing on the HO- 1 mRNA level at $4 \mathrm{~h}$. At $18 \mathrm{~h}$, the HUVEC PDK4 mRNA was significantly downregulated by quercetin in monoculture as well as co-culture $(77.4 \pm 2.8$ and $85.9 \pm 2.8 \%$, of the respective untreated controls), but because of the PDK4 upregulation in the co-culture $(182.8 \pm 3.3 \%)$, the absolute PDK4 levels in the quercetin-treated co-culture were signifi-
A
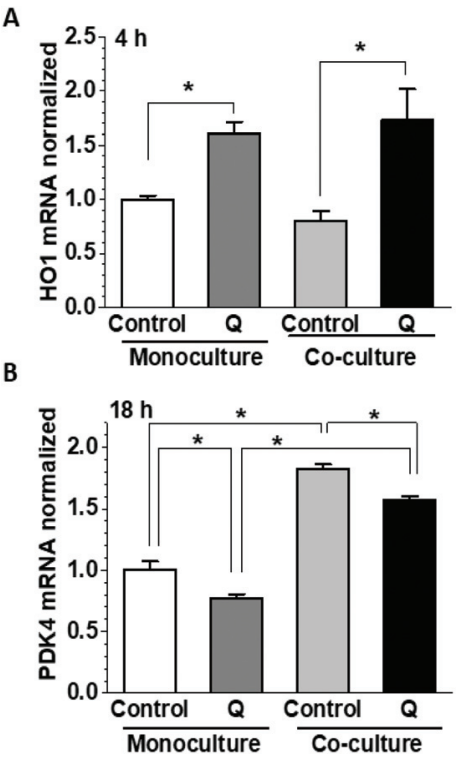

Fig. 7 Effect of HepG2 cells on gene expression in HUVEC. Gene expression changes in HUVEC cultured with or without HepG2 cells were measured in the presence or absence of $5 \mu \mathrm{M}$ quercetin. Confluent HUVEC and $80 \%$ confluent HepG2 cells were co-cultured for $1 \mathrm{~h}$ in $0.2 \%$ FBS EGM-2 medium on Transwells in parallel to control HUVEC. Medium with or without $5 \mu \mathrm{M}$ quercetin was then added to the apical side and the cells were further incubated for $4(A)$ or $18 \mathrm{~h}(\mathrm{~B})$. The mRNA expression of HO-1 (A) and PDK4 (B) was detected by ddPCR and normalized to the relevant monoculture control.

cantly higher ( 2-fold) than in monoculture (Fig. 7B). Thus, the effects elicited by co-culturing did not reverse, but rather modulated quercetin-induced changes in PDK4 expression, and did not significantly affect the short-term HO-1 upregulation.

\subsection{Glucose transport in HUVEC co-cultures}

To determine whether co-culture with HepG2 cells affects glucose uptake and transport by HUVEC, cells seeded on Transwells were cultured alone or with HepG2 cells in the presence or absence of $5 \mu \mathrm{M}$ quercetin on the apical side, and then $\mathrm{D}-\left[{ }^{14} \mathrm{C}(\mathrm{U})\right]$-glucose transport was measured. There were no changes in the monolayer integrity as measured by the TEER (Fig. 8A). Compared to the monoculture, HUVEC in co-culture incubated with $5 \mathrm{mM}$ glucose transported less $\mathrm{D}-\left[{ }^{14} \mathrm{C}(\mathrm{U})\right]$ glucose across the monolayer, and this effect was slightly alleviated in the presence of quercetin (Fig. 8B). No statistically significant effect was observed for the glucose uptake in the HUVEC or HepG2 cells. Depending on the role of different glucose transporters in the uptake, the effects could be modulated by the total glucose levels in the uptake assay and so we tested lower glucose concentration in the transport/uptake solution. When $0.5 \mathrm{mM}$ glucose was used in the HUVEC-HepG2 co-culture, quercetin pre-incubation significantly increased the glucose uptake in the HepG2 cells, but not in the HUVEC (Fig. 8C). At this concentration, the trans- 


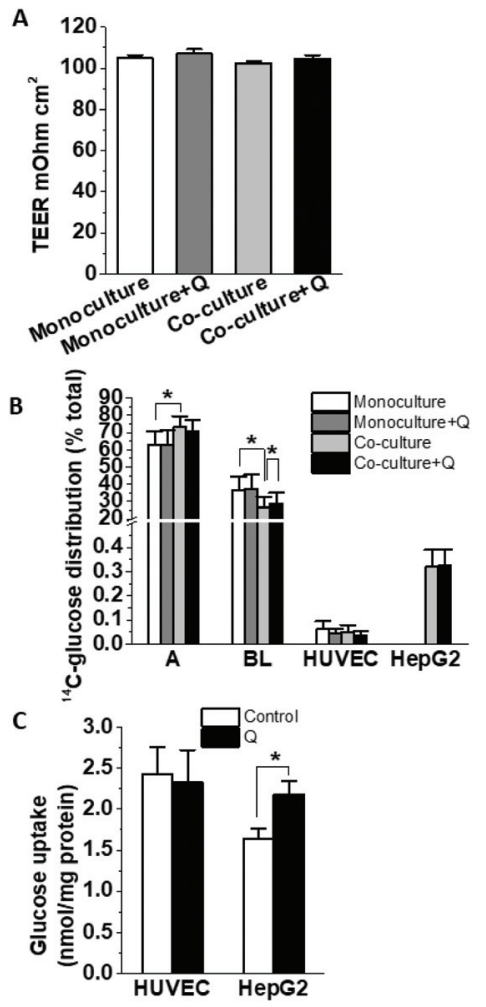

Fig. 8 The effect of quercetin on glucose uptake and transport in HUVEC and HepG2 cell co-cultures. Confluent HUVEC were incubated with or without HepG2 cells on the basolateral side for $1 \mathrm{~h}$, then medium with or without $5 \mu \mathrm{M}$ quercetin was added to the apical side and the cells were cultured for $18 \mathrm{~h}$. The HUVEC monolayer integrity was checked by measuring TEER (A). Glucose transport was estimated by applying $\left.5 \mathrm{mM} \mathrm{D-}-{ }^{14} \mathrm{C}(\mathrm{U})\right]$-glucose $\left(0.1 \mu \mathrm{Ci} \mathrm{ml^{-1 }}\right)$ on the apical side and after $10 \mathrm{~min}$, the distribution of ${ }^{14} \mathrm{C}$-label was measured in the apical, basolateral and cellular compartments (B). HUVEC-HepG2 cocultures treated for $18 \mathrm{~h}$ with or without $5 \mu \mathrm{M}$ quercetin were incubated for $10 \mathrm{~min}$ with $0.5 \mathrm{mM} \mathrm{D}-\left[{ }^{14} \mathrm{C}(\mathrm{U})\right]$-glucose on the apical side and the uptake for both HUVEC and HepG2 cells was determined from the radioactivity measured in cell lysates $(\mathrm{C})$.

port across the monolayer was not significantly affected (data not shown).

\section{Discussion}

Food-derived polyphenols have been studied as candidates for interventions to lower the risk of type 2 diabetes, based on their reported ability to regulate glucose absorption and metabolism and alleviate endothelial dysfunction. The mechanism of these activities has been explored through numerous in vitro, animal and human studies. In recent years, a limited number of co-culture cell models have been used to investigate some aspects of polyphenol metabolism and signalling. ${ }^{36}$ For example, co-cultures of Caco2 enterocytes and HT29-MTX goblet cells were used to study polyphenol transport and conjugation. ${ }^{37}$ Protective effects of polyphenols during inflammation have been elucidated in models combining macro- phages and gingival fibroblasts ${ }^{38}$ or adipocytes, ${ }^{39}$ or monocytes and Caco- 2 cells. ${ }^{40}$ Other studies explored anti-inflammatory potential of anthocyanins in co-cultures of gingival epithelial cells and fibroblasts ${ }^{41}$ or epithelial and endothelial cells. ${ }^{42,43}$ Recently, quercetin was shown to attenuate apoptosis of pancreatic $\beta$-cells through NO signalling in endothelial cells. ${ }^{44}$ However, our comprehension of the interplay between different cells in similar systems is still limited. Therefore, here we aimed to extend our previous study on the impact of quercetin on endothelial cell metabolism ${ }^{30}$ by including liver cells in the model, in order to examine the potential role of microenvironment and paracrine messengers. Different aspects of the interaction between endothelial and liver cells have been studied previously in organoids and systems combining endothelial cells with hepatocytes or HepG2 cells. ${ }^{45-47}$ HepG2 cells were shown to regulate lymphocyte transmigration across sinusoidal endothelial cells. ${ }^{48}$ Several studies addressed the communication via soluble factors including vesicles, ${ }^{49}$ although physical contact between HUVEC and HepG2 cells was required for angiogenic stimulation. ${ }^{50}$ In vivo, liver endothelial cells form a discontinuous, fenestrated layer, and are separated from the other liver cells by a thin Disse space, allowing for free exchange of soluble and other factors. Therefore, we have investigated the impact of liver cells on intact mature endothelial monolayers by utilizing a contactfree co-culture of endothelial and HepG2 cells and further studied the changes in the endothelial gene expression under the combined impact of co-culture and quercetin exposure. Because of the challenges associated with culturing primary hepatocytes, carcinoma-derived HepG2 cells were used as they are well characterized and afford better reproducibility. However, since there are metabolic differences between normal and transformed cells, it is possible that our results are more pertinent to tumour environment than normal tissue. We found that in the presence of HepG2 cells, HUVEC expressed increased levels of PDK4 mRNA and lower levels of GLUT1 and HO-1 protein. The HepG2 cells secreted TGF $\beta 1$ and dramatically increased lactate concentration in the medium compared to the HUVEC cultured alone. These and other soluble factors possibly contributed to the observed changes in gene expression, but continuous feedback from HepG2 cells was required for the maximum impact as HepG2conditioned medium had only limited effect. These were specific to the HepG2 cells as the only detected change in endothelial cells co-cultured with LHCN-M2 immortalized skeletal muscle cells was downregulation of GLUT1 protein. Neither cell line affected the tightness of the endothelial monolayer. Since endothelial HO-1 and PDK4 gene expression has been shown to be significantly affected by quercetin exposure after 4 and $18 \mathrm{~h}$ respectively, ${ }^{30}$ we looked at whether this is altered in the co-culture. HO- 1 mRNA was upregulated by $4 \mathrm{~h}$-exposure to quercetin both in monoculture and coculture. At the same time there were no significant differences between the HO-1 levels in the two settings in the absence of quercetin, most likely because the $4 \mathrm{~h}$-incubation was too short for the full effect of the co-culture to take place. 
PDK4 mRNA was upregulated in the co-culture after $18 \mathrm{~h}$, but quercetin decreased the mRNA levels in co-cultured HUVEC to the levels comparable to the untreated cells in monoculture. Thus, although the general impact of quercetin on endothelial expression of PDK4 and HO-1 was similar in endothelial monoculture and co-culture, the presence of HepG2 cells moderated the quercetin effects on PDK4, but not HO-1 expression.

Co-culturing with HepG2 cells decreased the amount of glucose transported across the HUVEC monolayer, possibly related to the GLUT1 downregulation. This was slightly alleviated by quercetin, consistent with our previous finding that quercetin could increase glucose transport in HUVEC. In addition, quercetin application to the apical side of endothelial cells stimulated glucose uptake in HepG2 cells on the basolateral side, but only for low glucose concentration, possibly as a downstream effect of quercetin-triggered signalling. At this quercetin concentration, the amount of the polyphenol transported to the basolateral side of the endothelial monolayer would be quite small, so an indirect effect through HUVEC is more plausible than quercetin acting on the HepG2 directly. Altered expression of glucose or drug transporters as well as changes in ROS and extracellular signalling in the endothelial cells could have a downstream effect on the adjacent HepG2 cells. Thus, in addition to direct effects, polyphenols such as quercetin could act on the target tissues through shifting the redox and metabolic balance in the endothelium and modifying the associated endothelial signalling to the underlying tissues.

\section{Conclusions}

In summary, we have used endothelial cells in co-culture with HepG2 cells as a more physiologically relevant configuration to study the impact of diet-derived polyphenol quercetin on their physiology. We have shown that the co-culture affects expression of various genes involved in metabolism (GLUT1 and PDK4) or redox balance (HO-1) in HUVEC, which can then contribute to regulating changes triggered by quercetin application, supporting the notion that the local environment in tissues has potential to modulate the impact of circulating molecules.

\section{Abbreviations}

ABCB1 ATP binding cassette subfamily B member 1

ABCC2 ATP binding cassette subfamily $\mathrm{C}$ member 2

ABCG2 ATP binding cassette subfamily G member 2

ApoE Apolipoprotein E

ddPCR Droplet digital PCR

DMEM Dulbecco's modified Eagle medium

EGM-2 Endothelial cell growth medium 2

eNOS Endothelial nitric oxide synthase

GLUT Glucose transporter

HIF1 $\alpha \quad$ Hypoxia induced factor $1 \alpha$
HO-1 Heme oxygenase-1

HPAE-PAD High performance anion exchange chromatography with pulsed amperometric detection

HUVEC Human umbilical vein endothelial cells

ICAM1 Intercellular adhesion molecule 1

MCT Monocarboxylate transporter

NO Nitric oxide

NOX NADPH oxidase

Nrf2 Nuclear factor erythroid 2-related factor 2

oxLDL Oxidized low density lipoprotein

PDK Pyruvate dehydrogenase kinase

ROS Reactive oxygen species

SIRT1 Sirtuin 1

TGF $\beta \quad$ Transforming growth factor beta

VCAM1 Vascular cell adhesion molecule 1

TBP TATA binding protein

TEER Transendothelial electrical resistance

\section{Author contributions}

ST and GW conceived and planned the project. ST carried out the experiments and analysed the data with $\mathrm{MJH}$ providing assistance with the muscle cell cultures. ST and GW wrote the manuscript and all authors provided feedback and contributed to the final version of the manuscript.

\section{Conflicts of interest}

The authors have no conflicts of interest to declare.

\section{Acknowledgements}

The presented research has been supported by funding from the European Research Council advanced grant number 322467 ('POLYTRUE?'). We thank Dr Vincent Mouly (Sorbonne Université, INSERM, Paris, France) for providing the immortalized skeletal muscle cells LHCN-M2.

\section{References}

1 V. Bauer and R. Sotnikova, Nitric oxide - the endotheliumderived relaxing factor and its role in endothelial functions, Gen. Physiol. Biophys., 2010, 29, 319-340.

2 M. J. Durand and D. D. Gutterman, Diversity in mechanisms of endothelium-dependent vasodilation in health and disease, Microcirculation, 2013, 20, 239-247.

3 H. F. Galley and N. R. Webster, Physiology of the endothelium, Br. J. Anaesth., 2004, 93, 105-113.

4 S. Rafii, J. M. Butler and B. S. Ding, Angiocrine functions of organ-specific endothelial cells, Nature, 2016, 529, 316-325.

5 J. G. Camp, K. Sekine, T. Gerber, H. Loeffler-Wirth, H. Binder, M. Gac, S. Kanton, J. Kageyama, G. Damm, D. Seehofer, L. Belicova, M. Bickle, R. Barsacchi, R. Okuda, 
E. Yoshizawa, M. Kimura, H. Ayabe, H. Taniguchi, T. Takebe and B. Treutlein, Multilineage communication regulates human liver bud development from pluripotency, Nature, 2017, 546, 533-538.

6 B. S. Ding, D. J. Nolan, J. M. Butler, D. James, A. O. Babazadeh, Z. Rosenwaks, V. Mittal, H. Kobayashi, K. Shido, D. Lyden, T. N. Sato, S. Y. Rabbany and S. Rafii, Inductive angiocrine signals from sinusoidal endothelium are required for liver regeneration, Nature, 2010, 468, 310315.

7 X. Pi, L. Xie and C. Patterson, Emerging Roles of Vascular Endothelium in Metabolic Homeostasis, Circ. Res., 2018, 123, 477-494.

8 A. Godecke and J. Haendeler, Intra- and Interorgan Communication in the Cardiovascular System: A Special View on Redox Regulation, Antioxid. Redox Signaling, 2017, 26, 613-615.

9 J. K. Freed and D. D. Gutterman, Communication Is Key: Mechanisms of Intercellular Signaling in Vasodilation, J. Cardiovasc. Pharmacol., 2017, 69, 264-272.

$10 \mathrm{~J}$. Keaney and M. Campbell, The dynamic blood-brain barrier, FEBS J., 2015, 282, 4067-4079.

11 T. Kubota, N. Kubota, H. Kumagai, S. Yamaguchi, H. Kozono, T. Takahashi, M. Inoue, S. Itoh, I. Takamoto, T. Sasako, K. Kumagai, T. Kawai, S. Hashimoto, T. Kobayashi, M. Sato, K. Tokuyama, S. Nishimura, M. Tsunoda, T. Ide, K. Murakami, T. Yamazaki, O. Ezaki, K. Kawamura, H. Masuda, M. Moroi, K. Sugi, Y. Oike, H. Shimokawa, N. Yanagihara, M. Tsutsui, Y. Terauchi, K. Tobe, R. Nagai, K. Kamata, K. Inoue, T. Kodama, K. Ueki and T. Kadowaki, Impaired insulin signaling in endothelial cells reduces insulin-induced glucose uptake by skeletal muscle, Cell Metab., 2011, 13, 294-307.

12 M. Konishi, M. Sakaguchi, S. M. Lockhart, W. Cai, M. E. Li, E. P. Homan, C. Rask-Madsen and C. R. Kahn, Endothelial insulin receptors differentially control insulin signaling kinetics in peripheral tissues and brain of mice, Proc. Natl. Acad. Sci. U. S. A., 2017, 114, E8478-E8487.

13 B. S. Ding, Z. Cao, R. Lis, D. J. Nolan, P. Guo, M. Simons, M. E. Penfold, K. Shido, S. Y. Rabbany and S. Rafii, Divergent angiocrine signals from vascular niche balance liver regeneration and fibrosis, Nature, 2014, 505, 97-102.

14 T. Schewe, Y. Steffen and H. Sies, How do dietary flavanols improve vascular function? A position paper, Arch. Biochem. Biophys., 2008, 476, 102-106.

15 K. Yamagata, M. Tagami and Y. Yamori, Dietary polyphenols regulate endothelial function and prevent cardiovascular disease, Nutrition, 2015, 31, 28-37.

16 N. Suganya, E. Bhakkiyalakshmi, D. V. Sarada and K. M. Ramkumar, Reversibility of endothelial dysfunction in diabetes: role of polyphenols, Br. J. Nutr., 2016, 116, 223-246.

17 T. Hussain, B. Tan, Y. Yin, F. Blachier, M. C. Tossou and N. Rahu, Oxidative Stress and Inflammation: What Polyphenols Can Do for Us?, Oxid. Med. Cell. Longevity, 2016, 2016, 7432797.
18 G. E. Mann, B. Bonacasa, T. Ishii and R. C. Siow, Targeting the redox sensitive Nrf2-Keap1 defense pathway in cardiovascular disease: protection afforded by dietary isoflavones, Curr. Opin. Pharmacol., 2009, 9, 139-145.

19 J. H. Kim, C. Auger and V. B. Schini-Kerth, Activation of eNOS by polyphenol-rich products and polyphenolic compounds, Curr. Pharm. Des., 2014, 20, 3521-3529.

20 C. H. Hung, S. H. Chan, P. M. Chu and K. L. Tsai, Quercetin is a potent anti-atherosclerotic compound by activation of SIRT1 signaling under oxLDL stimulation, Mol. Nutr. Food Res., 2015, 59, 1905-1917.

21 M. Sanchez, M. Galisteo, R. Vera, I. C. Villar, A. Zarzuelo, J. Tamargo, F. Perez-Vizcaino and J. Duarte, Quercetin downregulates NADPH oxidase, increases eNOS activity and prevents endothelial dysfunction in spontaneously hypertensive rats, J. Hypertens., 2006, 24, 75-84.

22 H. S. Jones, A. Gordon, S. G. Magwenzi, K. Naseem, S. L. Atkin and F. L. Courts, The dietary flavonol quercetin ameliorates angiotensin II-induced redox signaling imbalance in a human umbilical vein endothelial cell model of endothelial dysfunction via ablation of p47phox expression, Mol. Nutr. Food Res., 2016, 60, 787797.

23 Y. Shen, N. C. Ward, J. M. Hodgson, I. B. Puddey, Y. Wang, D. Zhang, G. J. Maghzal, R. Stocker and K. D. Croft, Dietary quercetin attenuates oxidant-induced endothelial dysfunction and atherosclerosis in apolipoprotein E knockout mice fed a high-fat diet: a critical role for heme oxygenase-1, Free Radical Biol. Med., 2013, 65, 908-915.

24 C. Li, W. J. Zhang, J. Choi and B. Frei, Quercetin affects glutathione levels and redox ratio in human aortic endothelial cells not through oxidation but formation and cellular export of quercetin-glutathione conjugates and upregulation of glutamate-cysteine ligase, Redox Biol., 2016, 9, 220228.

25 P. Galindo, I. Rodriguez-Gomez, S. Gonzalez-Manzano, M. Duenas, R. Jimenez, C. Menendez, F. Vargas, J. Tamargo, C. Santos-Buelga, F. Perez-Vizcaino and J. Duarte, Glucuronidated quercetin lowers blood pressure in spontaneously hypertensive rats via deconjugation, $P L O S$ One, 2012, 7, e32673.

26 A. Perez, S. Gonzalez-Manzano, R. Jimenez, R. Perez-Abud, J. M. Haro, A. Osuna, C. Santos-Buelga, J. Duarte and F. Perez-Vizcaino, The flavonoid quercetin induces acute vasodilator effects in healthy volunteers: correlation with beta-glucuronidase activity, Pharmacol. Res., 2014, 89, 1118.

27 S. Tribolo, F. Lodi, C. Connor, S. Suri, V. G. Wilson, M. A. Taylor, P. W. Needs, P. A. Kroon and D. A. Hughes, Comparative effects of quercetin and its predominant human metabolites on adhesion molecule expression in activated human vascular endothelial cells, Atherosclerosis, 2008, 197, 50-56.

28 C. Li, W. J. Zhang and B. Frei, Quercetin inhibits LPSinduced adhesion molecule expression and oxidant production in human aortic endothelial cells by p38-mediated 
Nrf2 activation and antioxidant enzyme induction, Redox Biol., 2016, 9, 104-113.

29 T. Koga and M. Meydani, Effect of plasma metabolites of $(+)$-catechin and quercetin on monocyte adhesion to human aortic endothelial cells, Am. J. Clin. Nutr., 2001, 73, 941-948.

30 S. Tumova, A. Kerimi and G. Williamson, Long term treatment with quercetin in contrast to the sulfate and glucuronide conjugates affects HIF1alpha stability and Nrf2 signaling in endothelial cells and leads to changes in glucose metabolism, Free Radical Biol. Med., 2019, 137, 158-168.

31 C. H. Zhu, V. Mouly, R. N. Cooper, K. Mamchaoui, A. Bigot, J. W. Shay, J. P. Di Santo, G. S. Butler-Browne and W. E. Wright, Cellular senescence in human myoblasts is overcome by human telomerase reverse transcriptase and cyclin-dependent kinase 4: consequences in aging muscle and therapeutic strategies for muscular dystrophies, Aging Cell, 2007, 6, 515-523.

32 M. J. Houghton, A. Kerimi, V. Mouly, S. Tumova and G. Williamson, Gut microbiome catabolites as novel modulators of muscle cell glucose metabolism, FASEB J., 2019, 33, 1887-1898.

33 J. F. Huggett, C. A. Foy, V. Benes, K. Emslie, J. A. Garson, R. Haynes, J. Hellemans, M. Kubista, R. D. Mueller, T. Nolan, et al., The digital MIQE guidelines: Minimum Information for Publication of Quantitative Digital PCR Experiments, Clin. Chem., 2013, 59, 892-902.

34 S. Tumova, A. Kerimi, K. E. Porter and G. Williamson, Transendothelial glucose transport is not restricted by extracellular hyperglycaemia, Vasc. Pharmacol., 2016, 87, 219-229.

35 Y. Okita, A. Kamoshida, H. Suzuki, K. Itoh, H. Motohashi, K. Igarashi, M. Yamamoto, T. Ogami, D. Koinuma and M. Kato, Transforming growth factor-beta induces transcription factors MafK and Bach1 to suppress expression of the heme oxygenase-1 gene, J. Biol. Chem., 2013, 288, 20658-20667.

36 C. Grootaert, S. Kamiloglu, E. Capanoglu and J. Van Camp, Cell Systems to Investigate the Impact of Polyphenols on Cardiovascular Health, Nutrients, 2015, 7, 9229-9255.

37 F. Jailani and G. Williamson, Effect of edible oils on quercetin, kaempferol and galangin transport and conjugation in the intestinal Caco-2/HT29-MTX co-culture model, Food Funct., 2014, 5, 653-662.

38 M. P. Morin and D. Grenier, Regulation of matrix metalloproteinase secretion by green tea catechins in a threedimensional co-culture model of macrophages and gingival fibroblasts, Arch. Oral Biol., 2017, 75, 89-99.

39 Y. Sakamoto, J. Kanatsu, M. Toh, A. Naka, K. Kondo and K. Iida, The Dietary Isoflavone Daidzein Reduces Expression of Pro-Inflammatory Genes through PPARalpha/ gamma and JNK Pathways in Adipocyte and Macrophage Co-Cultures, PLoS One, 2016, 11, e0149676.

40 H. Zhang, Y. I. Hassan, J. Renaud, R. Liu, C. Yang, Y. Sun and R. Tsao, Bioaccessibility, bioavailability, and antiinflammatory effects of anthocyanins from purple root veg- etables using mono- and co-culture cell models, Mol. Nutr. Food Res., 2017, 61, 1600928.

41 T. B. Lombardo Bedran, D. Palomari Spolidorio and D. Grenier, Green tea polyphenol epigallocatechin-3-gallate and cranberry proanthocyanidins act in synergy with cathelicidin (LL-37) to reduce the LPS-induced inflammatory response in a three-dimensional co-culture model of gingival epithelial cells and fibroblasts, Arch. Oral Biol., 2015, 60, 845-853.

42 S. Kuntz, H. Asseburg, S. Dold, A. Rompp, B. Frohling, C. Kunz and S. Rudloff, Inhibition of low-grade inflammation by anthocyanins from grape extract in an in vitro epithelial-endothelial co-culture model, Food Funct., 2015, 6, 1136-1149.

43 S. Kamiloglu, C. Grootaert, E. Capanoglu, C. Ozkan, G. Smagghe, K. Raes and J. Van Camp, Anti-inflammatory potential of black carrot (Daucus carota L.) polyphenols in a co-culture model of intestinal Caco-2 and endothelial EA.hy926 cells, Mol. Nutr. Food Res., 2017, 61, 1600455.

44 N. Suganya, K. P. Mani, D. Sireesh, P. Rajaguru, M. Vairamani, T. Suresh, T. Suzuki, S. Chatterjee and K. M. Ramkumar, Establishment of pancreatic microenvironment model of ER stress: Quercetin attenuates beta-cell apoptosis by invoking nitric oxidecGMP signaling in endothelial cells, J. Nutr. Biochem., 2018, 55, 142-156.

45 Y. B. Kang, S. Rawat, J. Cirillo, M. Bouchard and H. M. Noh, Layered long-term co-culture of hepatocytes and endothelial cells on a transwell membrane: toward engineering the liver sinusoid, Biofabrication, 2013, 5, 045008.

46 M. A. Guzzardi, F. Vozzi and A. D. Ahluwalia, Study of the crosstalk between hepatocytes and endothelial cells using a novel multicompartmental bioreactor: a comparison between connected cultures and cocultures, Tissue Eng., Part A, 2009, 15, 3635-3644.

47 Y. Nahmias, M. Casali, L. Barbe, F. Berthiaume and M. L. Yarmush, Liver endothelial cells promote LDL-R expression and the uptake of HCV-like particles in primary rat and human hepatocytes, Hepatology, 2006, 43, 257-265.

48 S. Edwards, P. F. Lalor, G. B. Nash, G. E. Rainger and D. H. Adams, Lymphocyte traffic through sinusoidal endothelial cells is regulated by hepatocytes, Hepatology, 2005, 41, 451-459.

49 F. Royo, L. Moreno, J. Mleczko, L. Palomo, E. Gonzalez, D. Cabrera, A. Cogolludo, F. P. Vizcaino, S. van-Liempd and J. M. Falcon-Perez, Hepatocyte-secreted extracellular vesicles modify blood metabolome and endothelial function by an arginase-dependent mechanism, Sci. Rep., 2017, 7, 42798.

50 G. G. Y. Chiew, A. Fu, K. Perng Low and K. Qian Luo, Physical supports from liver cancer cells are essential for differentiation and remodeling of endothelial cells in a HepG2-HUVEC co-culture model, Sci. Rep., 2015, 5, 10801. 